\title{
A Four-Dimensional Objective Analysis Scheme and Multitriangle Technique for Wind Profiler Data
}

\author{
PHILLIP L. SPENCER \\ NOAA/National Severe Storms Laboratory, Norman, Oklahoma \\ PAUL R. JANISH \\ NOAA/National Centers for Environmental Prediction, Storm Prediction Center, Norman, Oklahoma \\ Charles A. Doswell III \\ NOAA/National Severe Storms Laboratory, Norman, Oklahoma
}

(Manuscript received 28 February 1997, in final form 31 March 1998)

\begin{abstract}
A 4D Barnes objective analysis scheme for wind profiler data is developed in order to improve upon previously developed 2D analysis schemes. A significant shortcoming of the 2D schemes is their sensitivity to gaps in the profiler time-height series; they may produce unrealistic gradient information if large data-void regions are present. The 4D analysis scheme described herein, however, provides an effective means for dealing with data voids within profiler time-height series. The $4 \mathrm{D}$ analysis scheme differs from the $2 \mathrm{D}$ techniques in that data from neighboring profiler stations affect the time-height wind analysis at each site. This allows the analysis scheme to produce smooth, spatially and temporally consistent time-height wind analyses for each station of the Wind Profiler Network (WPN), even if large data gaps are present.

Gridded time-height wind fields at each profiler site resulting from the 4D analysis scheme are provided as input to a line integral-equivalent technique that is applied over many WPN triangles for the purpose of diagnosing the kinematic and thermodynamic structure of subsynoptic-scale weather systems. Time-height series of trianglederived variables have been proven elsewhere to be an effective method for diagnosing subsynoptic-scale temporal structure of weather systems; mapping the irregularly spaced triangle information onto a regular, quasihorizontal grid provides a complementary perspective of their spatial structure and evolution.
\end{abstract}

\section{Introduction}

Several observational platforms have become available to weather forecasters and researchers in recent years, allowing for better diagnoses of mesoscale and stormscale processes. For example, Doppler radar, atmospheric sounders aboard Geostationary Operational Environmental Satellites, and surface mesonetwork data have all contributed to our improved diagnostic capabilities (Brown and Wood 1991; Menzel and Purdom 1994; Barnes 1978). Similar opportunities are available via the high temporal resolution data provided by the Wind Profiler Network (WPN), which makes it possible to observe changes in subsynoptic-scale atmospheric structure throughout the troposphere (Carr et al. 1995; hereafter CSDP95). Although wind profilers do not pro-

Corresponding author address: Phillip L. Spencer, National Severe Storms Laboratory, 1313 Halley Circle, Norman, OK 73069.

E-mail: spencer@nssl.noaa.gov vide temperature and moisture information, hourly wind observations from the profiler network provide a temporal resolution of weather systems that is 12 times higher than that provided by the twice-daily rawinsonde observations.

The potential of wind profiler data for diagnosing the structure and evolution of subsynoptic-scale weather systems far exceeds simple examination of time-height series of raw observations at individual stations. As Doswell and Caracena (1988) point out, information about derivatives of the wind field (e.g., divergence) is at least as important as information concerning the wind field itself. Therefore, techniques for estimating wind derivatives from profiler data have been developed for the purpose of studying kinematic and thermodynamic structure of mesoscale convective systems (Yoe et al. 1992), snowstorms (Carlson and Forbes 1989), sharp troughs in the baroclinic westerlies (Bluestein and Speheger 1995), amplifying and decaying baroclinic waves (Spencer et al. 1996; hereafter SCD96), upper-tropospheric fronts and jet streams (Neiman and Shapiro 
1989), and tropopause folds (Karyampudi et al. 1995), for example. Since a complete time-height series of wind observations from stations involved in line integral (Bellamy 1949; Ceselski and Sapp 1975) or linear vector point function (LVPF; Zamora et al. 1987; Doswell and Caracena 1988; Davies-Jones 1993) methods is not always present, owing to missing data [either by failure to reach hourly "consensus" (Strauch et al. 1984) or by rejection via quality control algorithms], various schemes have been adopted to fill data voids. In addition, it is desirable that these hole-filling schemes provide a mechanism for substantially reducing unwanted scales in the data as well as lessening the impacts of instrument errors.

Linear interpolation has been a popular choice for filling data gaps, whereas Shuman filters (1957) and Gaussian weighting functions have been used to damp unwanted scales (Zamora et al. 1987; Neiman and Shapiro 1989; Hermes 1991; Karyampudi et al. 1995). CSDP95 discuss two objective analysis schemes for profiler time-height data that allow: 1) data gaps to be filled by either of the $2 \mathrm{D}$ schemes rather than by independent one-dimensional interpolation schemes applied in time and height, and 2) the data to be filtered simultaneously in time and height.

A theme common to the schemes mentioned above is that they are 2D; that is, data from each profiler timeheight series are analyzed onto a regular time-height grid independent of data from neighboring stations. The advantage is that station analyses can be performed quickly and with relative simplicity. A disadvantage is that these schemes demand a rather complete timeheight series for each station involved in subsequent triangle calculations. Large data voids present a challenge for 2D objective analysis schemes such as those developed by CSDP95 in that unrealistic velocity gradients in the analyses may be produced if analysis parameters are chosen haphazardly. Specifically, choosing parameters such that the analysis closely fits the observations forces spurious gradient information into the data voids (Barnes 1994). With this in mind, we desire an analysis scheme that not only damps unwanted scales, but also fills data voids without creating unrealistic velocity gradients.

The approach that we have developed extends the analysis scheme used by CSDP95 to include data from neighboring sites into each station's time-height analysis (as in Doswell 1977). Therefore, as we will show, missing data within a station's time series of observations do not pose as serious a threat as they would if a 2D time-height analysis scheme were used. ${ }^{1}$ Our 4D analysis scheme produces smooth, temporally and spatially consistent wind analyses at each site of the WPN. These analyses are used as input for a line integral-

\footnotetext{
${ }^{1}$ We note, however, that there is no substitute for adequate data.
}

equivalent technique that, when applied over many profiler triangles, provides a coherent picture of the evolution as well as the horizontal and vertical distribution of kinematic and thermodynamic variables within weather systems. This multitriangle technique provides a practical application of calculating derivatives directly from the irregularly distributed observing network, as suggested by Schaefer and Doswell (1979) and Doswell and Caracena (1988), rather than the traditional approach of using finite differences applied to a regular grid of the velocity components. These studies suggest that more accurate results are obtained when the firstorder wind derivatives are calculated directly from the data.

In the following section, we review the technique used by CSDP95 and SCD96 for deriving time-height series of kinematic and thermodynamic variables from a triangle of profilers, including the $2 \mathrm{D}$ objective analysis scheme used to produce filtered, gridded timeheight wind fields at profiler sites. In addition, we discuss the limitations of the 2D technique. Section 3 introduces a 4D objective analysis scheme for wind profiler data and provides tests of the scheme using both observations and an analytic wind field. Also included is a description of how triangle centroid information from many triangles is used to produce time series of quasi-horizontal displays. Section 4 contains a brief case study illustration of how wind profiler data are used to diagnose subsynoptic-scale processes associated with a winter weather event. Finally, section 5 provides a brief summary and concluding remarks.

\section{Two-dimensional technique: A review}

\section{a. Quality control and objective analysis}

The hourly time-height series of profiler winds are initially subjected to median and vertical shear checks similar to those developed by Brewster and Schlatter (1986) for the purpose of eliminating erroneous data. The median check searches for outliers in the data by comparing the wind components of each datum to those of a set of neighbors within the time-height section. The vertical shear check eliminates outliers by finding those data within a profile that are responsible for excessive vertical speed and/or directional shear. A complete description of the quality control scheme is found in CSDP95. In addition, hand-editing of erroneous wellcorrelated data (which normally pass quality control checks) is sometimes necessary.

Following CSDP95, the quality controlled timeheight series of observations then are used to produce a regular time-height grid $(200 \mathrm{~m} \times 1 \mathrm{~h})$ of the horizontal winds at each of three profiler sites. Each timeheight analysis is obtained by using one pass of a distance-dependent weighted averaging objective analysis scheme in which a datum's influence upon a gridpoint value $\left(g_{i}\right)$ is related to the vertical and temporal sepa- 
ration ( $R_{z}$ and $R_{t}$, respectively) of the datum $\left(f_{k}\right)$ and the grid point. Specifically, each gridpoint value is determined by

$$
g_{i}=\frac{\sum_{k=1}^{m} w_{i k} f_{k}}{\sum_{k=1}^{m} w_{i k}},
$$

where the weights $\left(w_{i k}\right)$ are determined by

$$
w_{i k}=\exp \left(-\frac{R_{z}^{2}}{\lambda^{2}}-\frac{R_{t}^{2}}{\tau^{2}}\right) .
$$

The parameters $\lambda$ and $\tau$ are smoothing parameters that determine how smooth the analysis will be; that is, values for $\lambda$ and $\tau$ control the response characteristics of the analysis scheme. The smoothing parameters often are defined as multiples of the average data separation (Koch et al. 1983). Let $\lambda=c_{1} \Delta z$ and $\tau=c_{2} \Delta t$, where $\Delta z$ is the average vertical data spacing (typically 250 $\mathrm{m})$ and $\Delta t$ the average temporal data spacing (typically $1 \mathrm{~h})$. For the analyses presented by CSDP95 and SCD96, $c_{1}=c_{2}=1.35$ so that the response at the Nyquist wavelength and period was nearly zero, whereas the response at three times the Nyquist interval was $e^{-1}$.

Factors influencing the subjectively chosen smoothing parameters should include the accuracy and representativeness of the data, data distribution characteristics (Doswell and Lasher-Trapp 1997), and the desired scales to resolve (Barnes 1973). We emphasize that for this $2 \mathrm{D}$ analysis scheme, data from neighboring profiler sites have no influence upon a station's analysis. For example, data from only the Vici, Oklahoma, profiler influences the Vici time-height analysis; data from the Lamont, Oklahoma, profiler (or any other station) have no impact upon the Vici analysis.

\section{b. Derivation of kinematic quantities}

Kinematic and thermodynamic fields can be derived directly from a small array (such as a triangle) of wind observation sites using, for example, the LVPF method (Zamora et al. 1987). Davies-Jones (1993) has shown that the LVPF method is essentially identical to any integral method that assumes linearity between sample points. Basically, the LVPF method decomposes the horizontal wind field (in our case, height-coordinate profiler winds interpolated onto pressure surfaces using a standard atmospheric sounding) into its linear components via a Taylor series expansion. Knowledge of the location of three noncolinear wind profiler sites closes a system composed of six equations and six unknowns, which is easily solved to yield the kinematic quantities. This technique provides time-height series estimates of vorticity and divergence, for example, that are assumed valid at triangle centroids.

The LVPF results are used to derive vertical motion

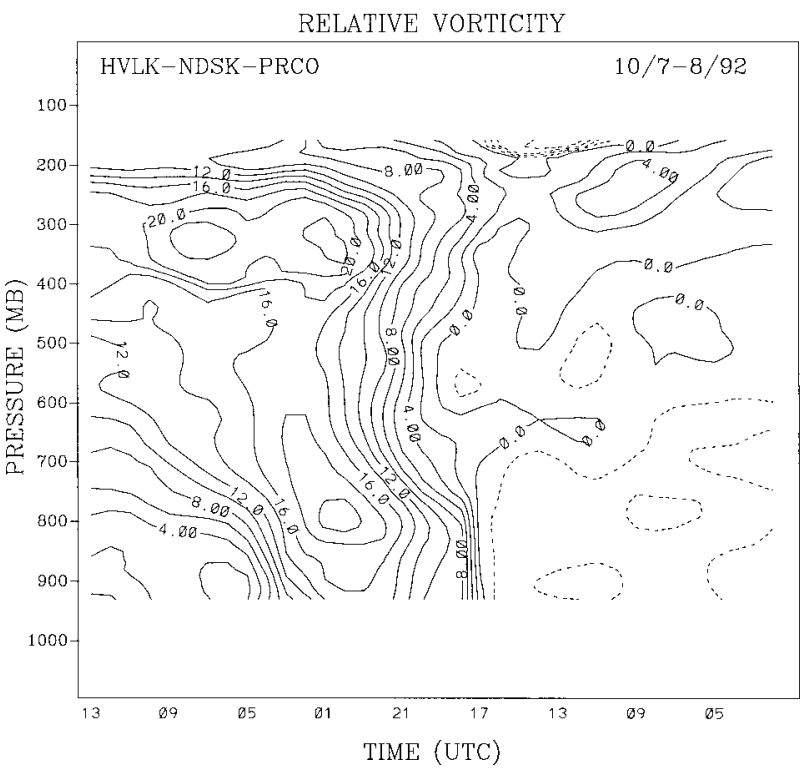

Fig. 1. Relative vorticity $\left(\times 10^{5} \mathrm{~s}^{-1}\right)$ derived from the Haviland, Kansas (HVLK), Neodesha, Kansas (NDSK), and Purcell, Oklahoma (PRCO), profiler data for the 36-h period ending 1300 UTC 8 Oct 1992. The field was derived via the $2 \mathrm{D}$ analysis scheme where $c_{1}=$ $c_{2}=1.35$. Contour interval is $2 \mathrm{~s}^{-1}$. Dashed contours indicate negative values for all plots. Time increases from right to left on all profiler time series plots.

estimates via the kinematic method in the manner described by SCD96 wherein "a linear adjustment procedure, which assumes that errors in the divergence are independent of pressure and that the vertical velocity is zero at both the top and bottom of the domain, is applied." We then calculate an "adjusted divergence" field, which is the divergence field that, when integrated, yields the "adjusted omega" field. All subsequent discussions involving divergence refer to the adjusted divergence field.

\section{c. Limitations}

We will now describe the limitations of the 2D technique that provided impetus for the development of the 4D technique. Consider the upstream-tilted baroclinic wave shown in Fig. 1. ${ }^{2}$ The raw time-height series data from each of the three stations contain only small data voids (not shown). To illustrate the impact that large areas of missing data have on the vorticity field derived via the 2D technique, five profiles (2000 UTC 7 October-0000 UTC 8 October) are removed from each of the three stations' time-height series of raw observations (Fig. 2). Rather than producing a gradual transition from lower to higher vorticity values from, say, 1800 UTC 7 October to 0100 UTC 8 October (as in Fig. 1),

\footnotetext{
${ }^{2}$ This case has been studied by Spencer et al. (1996).
} 


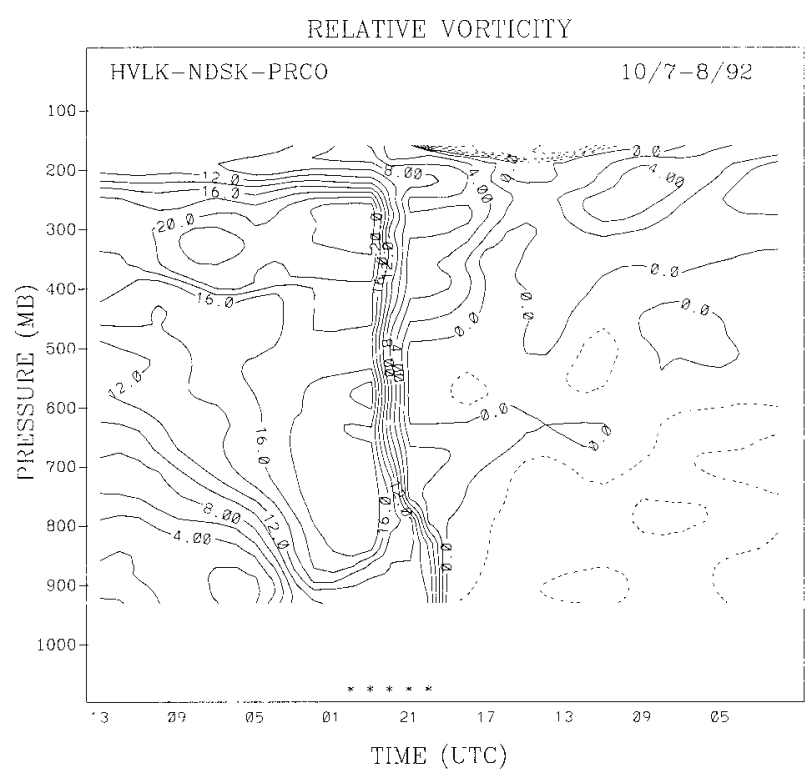

FIG. 2. Same as Fig. 1 except that the wind profiles from the raw time-height series for the period 2000 UTC 7 Oct-0000 UTC 8 Oct (indicated by asterisks) were deleted from each of the three stations.

the $2 \mathrm{D}$ analysis scheme concentrates the gradient information inside the data void, an undesirable result but one that is, nevertheless, consistent with the findings of Barnes (1994). Given the occasional occurrence of large vertical and temporal gaps in raw profiler time-height data, unrealistic gradient information can be expected from the $2 \mathrm{D}$ technique. Increasing the objective analysis shape parameters may relax the spurious gradients, but necessarily at the expense of a reduced capability for resolving details elsewhere in the time-height series.

Another limitation of the 2D technique involves its inability to provide a coherent picture of the horizontal structure of weather systems. The 2D technique was developed to provide information concerning kinematic and thermodynamic structure of weather systems as they pass through specific locations (e.g., triangle centroids). It does not combine centroid information derived from many triangles.

\section{Four-dimensional technique}

\section{a. Description of the objective analysis scheme and tests with real data}

The 4D objective analysis scheme is similar to the 2D scheme in that it uses distance-dependent weighted averaging, but the difference is the dimensionality of the weighting function. The weights for the 4D analysis scheme are determined by

$$
w_{i k}=\exp \left(-\frac{R_{z}^{2}}{\lambda^{2}}-\frac{R_{t}^{2}}{\tau^{2}}-\frac{R_{x y}^{2}}{\beta^{2}}\right),
$$

where the last term of the exponential allows data from neighboring profilers to be incorporated. Here, $R_{x y}$ rep- resents the horizontal distance between a datum and grid point and $\beta$ is a standard, Barnes-type smoothing parameter. We define $\beta$ as a multiple of the average station separation $(\Delta n$; approximately $270 \mathrm{~km})$ such that $\beta=$ $c_{3} \Delta n$. The constant $c_{3}$ is user-selectable and should be chosen according to the factors mentioned in section $2 \mathrm{a}$. We note that as $\beta$ approaches zero, the results of the $4 \mathrm{D}$ analysis scheme approach those of the $2 \mathrm{D}$ scheme.

Again consider the 7-8 October 1992 case in which the 2000 UTC 7 October-0000 UTC 8 October profiles are eliminated from the Haviland, Neodesha, and Purcell raw time-height series data. Shown in Fig. 3 are relative vorticity time-height fields derived using the 4D analysis scheme and the LVPF method. For each field, $c_{1}$ $=c_{2}=1.35$ (as in the examples presented in section $2 c)$. The difference in the fields is determined by the value of $c_{3}$ used in the 4D objective analysis scheme. The values of $c_{3}$ for Figs. 3a-d are 0.01, 0.50, 0.75, and 1.0 , respectively. With the parameter $c_{3}$ at our disposal, the degree of impact that data from neighboring sites have on each station's analysis can be controlled. As $c_{3}$ is increased, the impact becomes greater. As expected, a very small value of $c_{3}$ (e.g., 0.01; Fig. 3a) generates an analysis very similar to that produced by the $2 \mathrm{D}$ technique (Fig. 2) in which spurious gradient information is created within the data void.

Figure $3 \mathrm{~b}$ shows that when the value of $c_{3}$ is increased only modestly (yet is still exhibiting a relatively small value), the spurious gradient that was characteristic of the $2 \mathrm{D}$ technique is dramatically reduced. As $c_{3}$ is increased further (Figs. 3c,d), continued reduction of the gradient is accomplished as well as a dampening of the vorticity maxima, as expected. Therefore, by using a 4D analysis scheme that incorporates data from neighboring profilers, the negative impact of missing data on the wind analyses can be reduced.

The response function $(D)$ corresponding to (3) is

$$
D=\exp \left[-\lambda^{2}\left(\frac{\pi}{L_{z}}\right)^{2}-\tau^{2}\left(\frac{\pi}{L_{t}}\right)^{2}-\beta^{2}\left(\frac{\pi}{L_{x y}}\right)^{2}\right],
$$

where

$$
\begin{aligned}
L_{z} & =\text { vertical wavelength, } \\
L_{t} & =\text { period, } \\
L_{x y} & =\text { horizontal wavelength. }
\end{aligned}
$$

For the case study example presented in section 4 , the analysis parameters have been chosen to be $c_{1}=c_{2}=$ $c_{3}=1.15$, so that amplitudes of waves smaller than those that might be considered "marginally sampled" (Doswell and Caracena 1988) are damped considerably. The response for the Nyquist interval in any particular dimension is $\ll 10 \%$ (Fig. 4). In addition, vertical, temporal, and horizontal cutoff radii of $4 \lambda, 4 \tau$, and $4 \beta$, respectively, have been used. These are well within the guidelines suggested by Pauley and Wu (1990). 

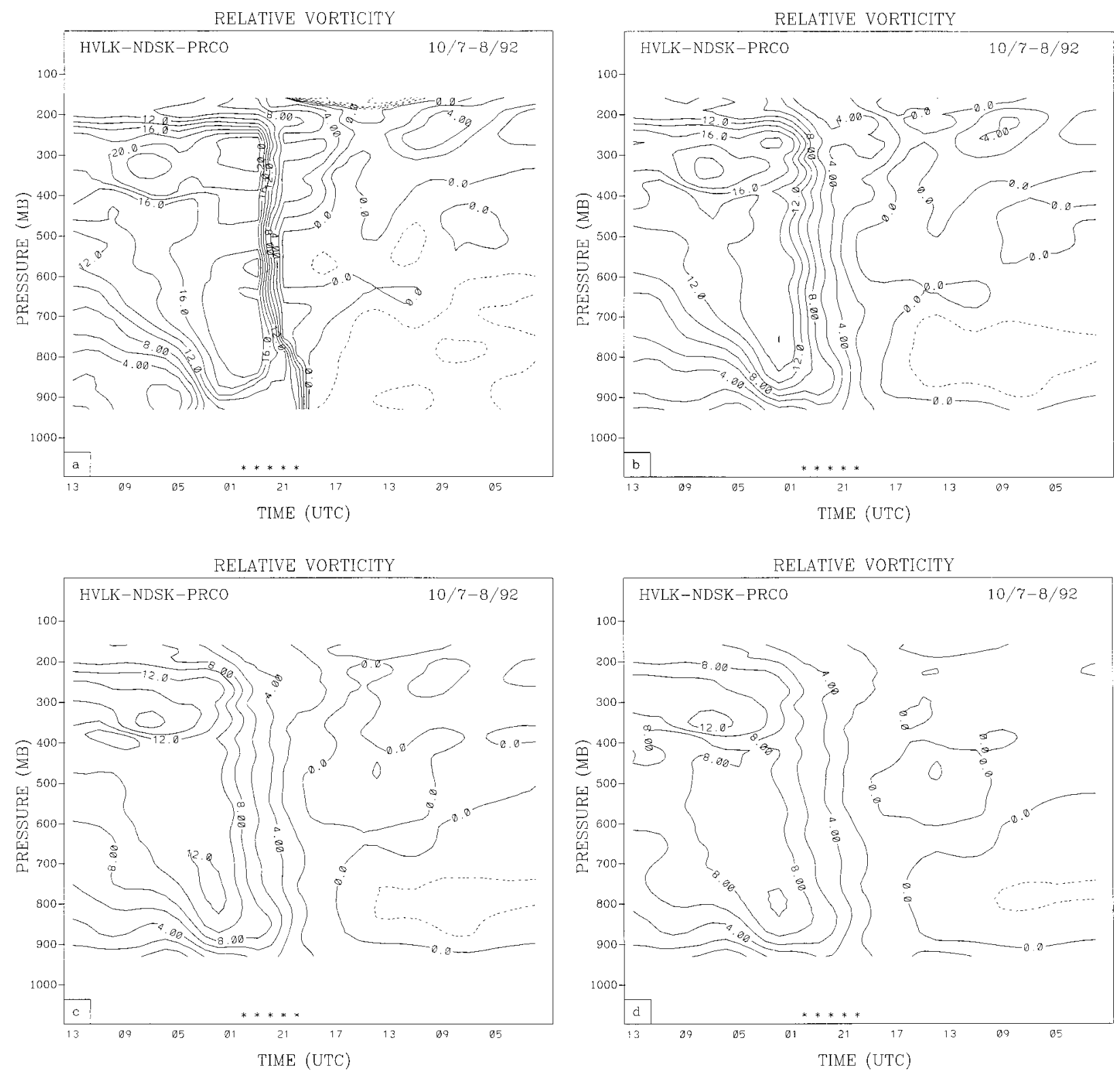

FIG. 3. Same as Fig. 2 except that the relative vorticity fields were derived via the $4 \mathrm{D}$ analysis scheme where (a) $c_{3}=0.01,(\mathrm{~b}) c_{3}=$ 0.50 , (c) $c_{3}=0.75$, and (d) $c_{3}=1.0$. For all fields, $c_{1}=c_{2}=1.35$.

\section{b. Objective analysis tests using an analytic wind field}

Consider an analytic horizontal wind field defined by the following:

$$
\begin{aligned}
& u(x, y)=-A \cos \left[\frac{2 \pi}{L}(x-c t)\right] \sin \left[\frac{2 \pi}{L} y\right] \\
& v(x, y)=A \sin \left[\frac{2 \pi}{L}(x-c t)\right] \cos \left[\frac{2 \pi}{L} y\right],
\end{aligned}
$$

where $A$ is the amplitude, $L$ is the wavelength, $c$ is the wave speed, and $t$ is time. Equation (5) represents eastward traveling waves of alternating cyclonic and anticyclonic flow patterns (Fig. 5). The 36-h time series of the $u$ component of the analytic wind field at station LMNO is shown as the thick curve in Fig. 6. To simulate missing data, five consecutive hours of data are removed from the LMNO time series (denoted by the asterisks along the abscissa in Fig. 6). Two Barnes analysis schemes are then used to produce a complete, hourly time series at LMNO. The first scheme uses a weight function in which data from neighboring stations are not included (NONEIGHBOR); specifically, 
(a)

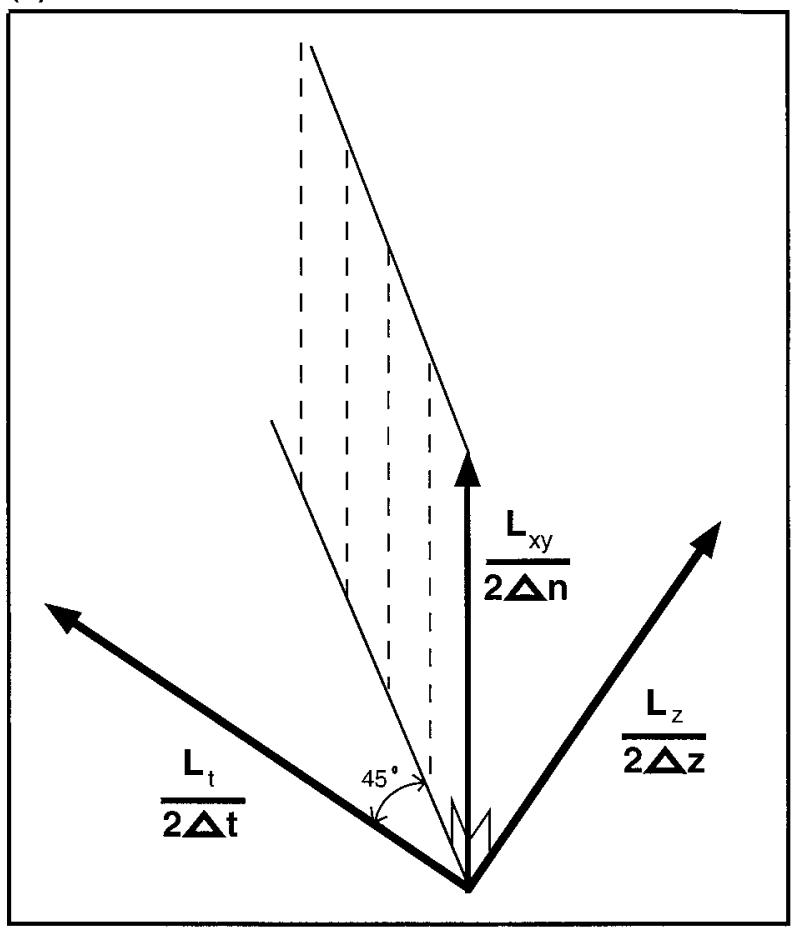

(b)

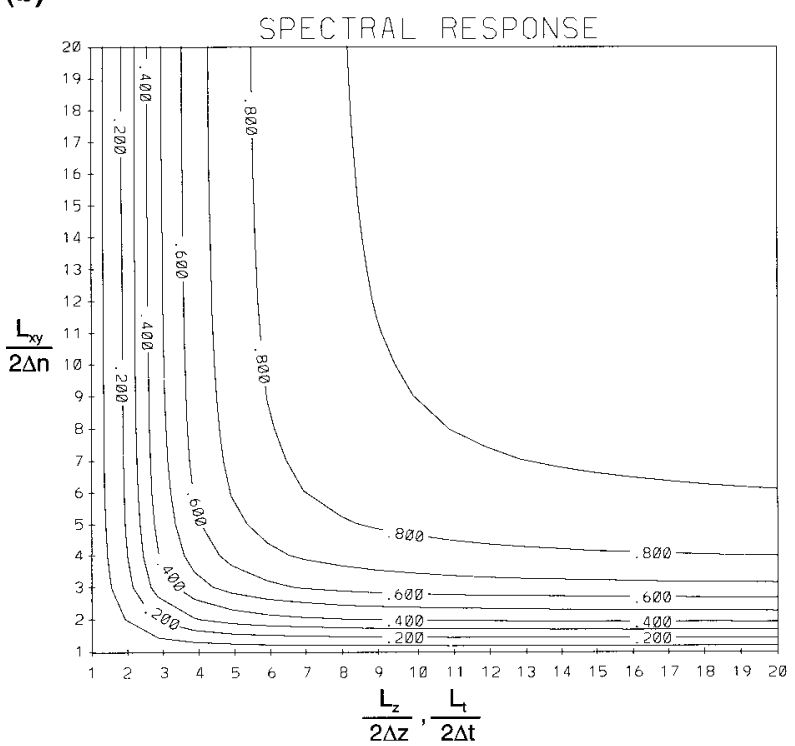

FIG. 4. Depiction of the 2D plane (Fig. 4a) along which the response function corresponding to the $4 \mathrm{D}$ weighting function is plotted (Fig. $4 \mathrm{~b})$. The shape coefficients used to produce Fig. $4 \mathrm{~b}$ are $c_{1}=c_{2}=$ $c_{3}=1.15$. The axes in Figs. 4a,b represent multiples of the Nyquist interval.

$$
w_{i k}=\exp \left(-\frac{R_{t}^{2}}{\tau^{2}}\right),
$$

where the parameters are as defined as in section $2 \mathrm{a}$ and $c_{2}=1.15$. This scheme is analogous to that described

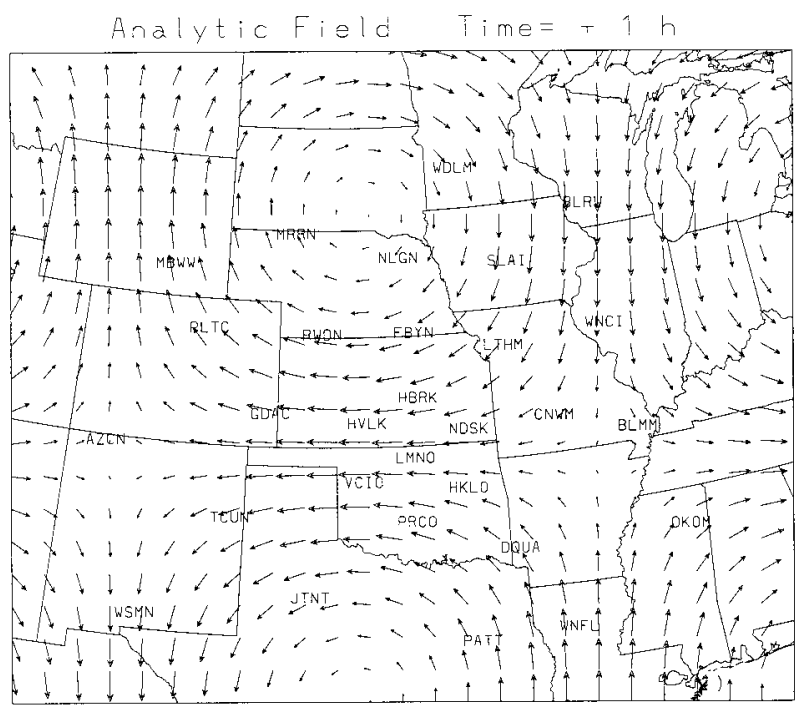

FIG. 5. Depiction of the analytic wind field defined by Eq. (5) for values of $A=40 \mathrm{~m} \mathrm{~s}^{-1}, L=3 \times 10^{6} \mathrm{~m}, c=20 \mathrm{~m} \mathrm{~s}^{-1}$, and $t=1 \mathrm{~h}$. Profiler locations are indicated by the four-letter identifiers.

in section $2 \mathrm{a}$, except that vertical variability is not considered. The second analysis scheme uses a weight function in which data from neighboring stations are included (NEIGHBOR); specifically,

$$
w_{i k}=\exp \left(-\frac{R_{t}^{2}}{\tau^{2}}-\frac{R_{x y}^{2}}{\beta^{2}}\right),
$$

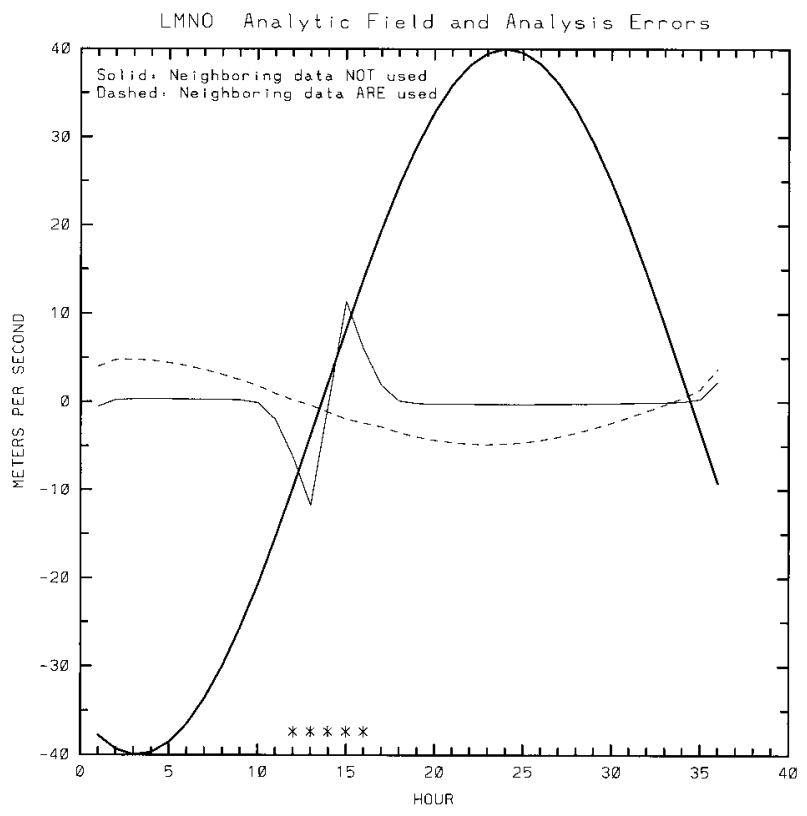

FIG. 6. Time series of the $u$ component of velocity $\left(\mathrm{m} \mathrm{s}^{-1}\right.$ ) corresponding to (5) for station LMNO (thick, solid curve). Analysis errors (analysis minus analytic values) resulting when five consecutive hours are removed from the time series (indicated by the asterisks) for the NONEIGHBOR and NEIGHBOR analyses are shown as the thin solid and dashed curves, respectively. See text for details. 


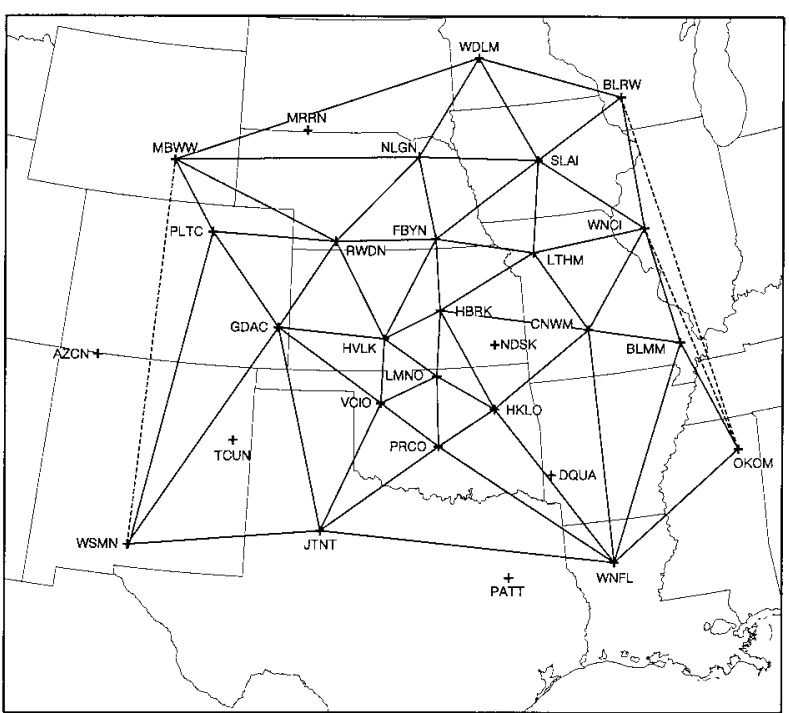

FIG. 7. Triangular tessellation for the 14-16 Feb 1993 case study. Stations excluded from the tessellation contained no data during the period. Dashed lines denote triangles eliminated from consideration.

where the parameters are as defined as in section $3 \mathrm{a}$ and $c_{2}=c_{3}=1.15$. This scheme is analogous to that described in section $3 \mathrm{a}$, except that vertical variability again is not considered. The analysis errors (analysis minus analytic values) for the NONEIGHBOR and NEIGHBOR schemes are shown as the thin solid and dashed curves, respectively, in Fig. 6.

Figure 6 clearly illustrates that the NEIGHBOR analysis scheme is the superior of the two schemes within the data void region. Mean absolute errors from this scheme average $1.2 \mathrm{~m} \mathrm{~s}^{-1}$ within the data void, whereas errors from the NONEIGHBOR analysis average $7.1 \mathrm{~m}$ $\mathrm{s}^{-1}$ for the same 5-h period. In the data-rich area of the extrema, the NEIGHBOR analysis damps the amplitude by approximately $12 \%$, whereas the NONEIGHBOR analysis provides a close fit to the observations. Therefore, incorporating data from neighboring stations into the analysis scheme provides an effective mechanism for hole-filling at the expense of a slight reduction in wave amplitude. This damping effect of the NEIGHBOR analysis can be mitigated by choosing a smaller smoothing parameter $\beta$. For example, when $\beta=0.75 \Delta n$ (not shown), the errors in the NEIGHBOR analysis are reduced by about $50 \%$ over those depicted in Fig. 6 . We again emphasize that for practical applications, the accuracy and representativeness of the data, data distribution characteristics, and the desired scales to resolve should guide the choice of shape parameter values.

\section{c. Multitriangle analysis}

The technique for deriving time-height series of kinematic variables from wind analysis produced via the 4D analysis scheme is identical to that described in section $2 \mathrm{~b}$. However, given that the 4D analysis scheme

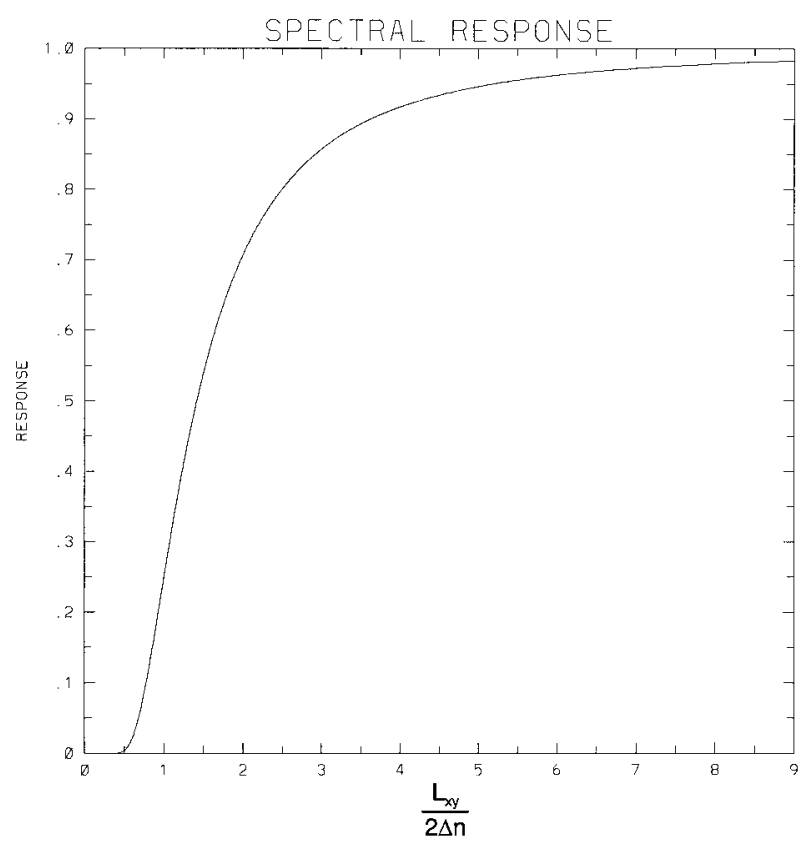

FIG. 8. Response function corresponding to the weighting scheme used to map centroid values onto a regular grid. The shape parameter $\alpha=0.75 \Delta n$. The abscissa is a dimensionless wavelength and represents multiples of the Nyquist interval.

produces gridded time-height winds at each profiler site whether or not it has substantial data voids, the LVPF method can be used for a large number of triangles.

Triangles are defined from the 29-station central United States Wind Profiler Network by the Delauney triangulation method described by Ripley (1981). One undesirable characteristic of the Delauney triangulation is that it creates a convex hull for the station set, so that excessively obtuse triangles often are produced along the boundaries. In this application, such triangles simply are eliminated from consideration. Normally, one or more stations has a totally or partially incomplete timeheight series of raw observations. Those stations with absolutely no data are completely ignored and the triangulation is performed without them (Fig. 7).

Centroid estimates of kinematic variables not only provide a useful means of diagnosing atmospheric structure via time-height sections, but when mapped onto a uniform horizontal display grid, they provide information about the horizontal structure and evolution of weather systems that move through the WPN. This mapping is accomplished by applying the weight function

$$
w_{i k}=\exp \left(-\frac{R_{x y}^{2}}{\alpha^{2}}\right),
$$

where the shape parameter $\alpha=0.75 \Delta n$ and $\Delta n, R_{x y}$ are as before. The response function corresponding to (8) is shown in Fig. 8 and indicates that $25 \%$ of the amplitude of the $2 \Delta n$ wave (Nyquist wavelength) is retained by this analysis scheme. Since the desired spectral 


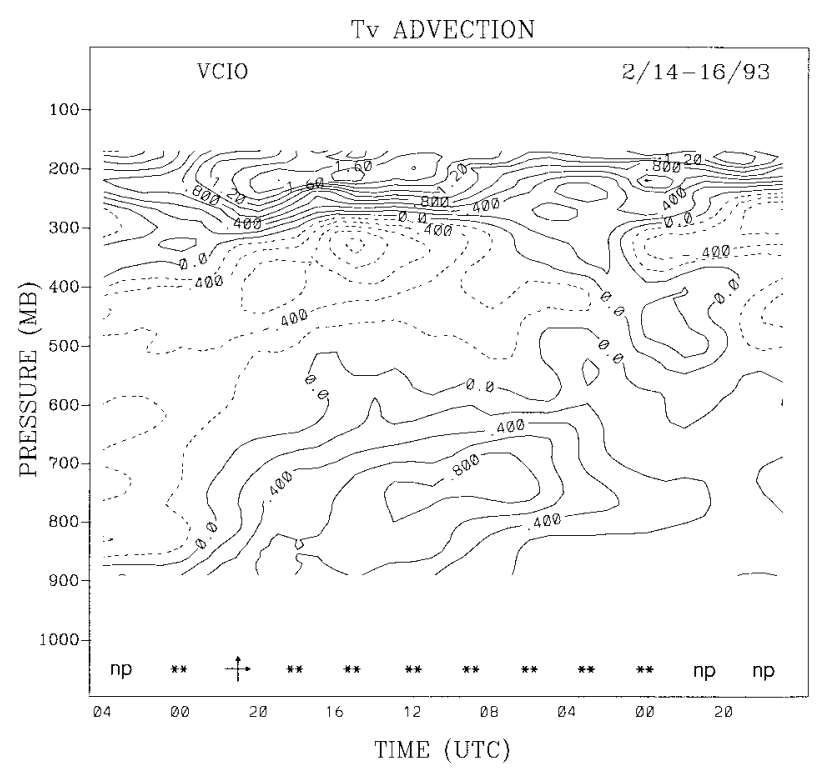

FIG. 9. Virtual temperature advection $\left(\mathrm{K} \mathrm{h}^{-1}\right)$ derived from the VCIO profiler for the 36-h period ending 0400 UTC 16 Feb 1993. Contour interval is $0.2 \mathrm{~K} \mathrm{~h}^{-1}$. Three-hourly current weather for GAG, which is approximately $40 \mathrm{~km}$ west-northwest of VCIO is also plotted. Here "np" indicates that no precipitation was reported.

filtering of the profiler winds already has been accomplished via (3), the choice of a light smoothing for this analysis is justified. The only purpose of this analysis is to map the irregularly distributed triangle centroid estimates of kinematic variables onto a uniform display grid. However, this analysis does smooth the noise created by the "roughing" effect (increasing amplitude at high wavenumber) inherent in derivative calculations.

The thermal structure of baroclinic systems is investigated by estimating the horizontal temperature gradient and thermal advection using the single-station technique described by Neiman and Shapiro (1989), in which the geostrophic thermal wind equation is applied to the station wind analyses. After time-height sections of thermal gradient and advection estimates are produced at each station, they are mapped onto a uniform horizontal grid via (8) to yield plan-view displays. Further details of the single-station technique including its limitations are found in Neiman and Shapiro (1989).

\section{Case study illustration: 14-16 February 1993}

A strong winter storm moved across the southern plains during 14-16 February 1993, producing a large band of snow accumulations greater than $20 \mathrm{~cm}$ across southern Kansas and northern Oklahoma into much of Missouri and northern Arkansas. Local snowfall amounts were as high as $75 \mathrm{~cm}$ near the northern Arkansas border. North of the heavy snowband, a large area of light snow fell, while heavy rain fell south of the band.

Profiler analyses indicate increasing low-level warm thermal advection over west-central Oklahoma by 2000
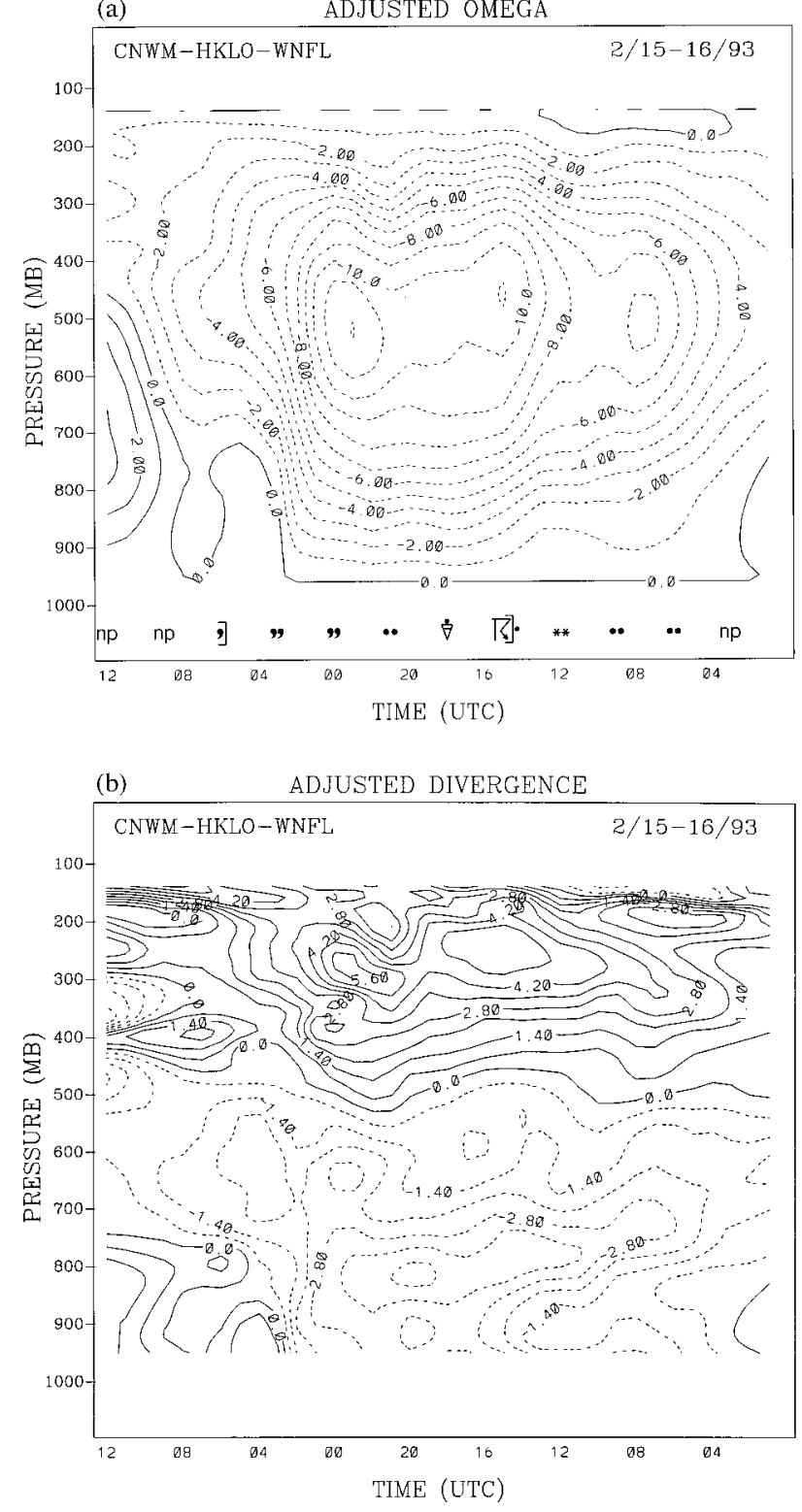

FIG. 10. CNWM-HKLO-WNFL profiler triangle calculations for the 36-h period ending 1200 UTC 16 Feb 1993 of (a) vertical motion (adjusted kinematic omega; contour interval of $1 \mu \mathrm{b} \mathrm{s}^{-1}$ ) and (b) adjusted divergence $\left(\times 10^{5} \mathrm{~s}^{-1}\right.$; contour interval of $\left.0.7 \mathrm{~s}^{-1}\right)$. Threehourly current weather for FSM, which lies just northwest of the triangle centroid, is also plotted in (a). Here "np" indicates that no precipitation was reported.

UTC 14 February (Fig. 9) in advance of a developing surface low. Precipitation developed in this area shortly after 2200 UTC and gradually expanded in coverage and intensity as deep upward motion ensued (not shown) and low-level warm thermal advection increased. Precipitation began to diminish in coverage over western Oklahoma by 2200 UTC 15 February as both rising motion and low-level warm thermal advection rapidly decreased. 


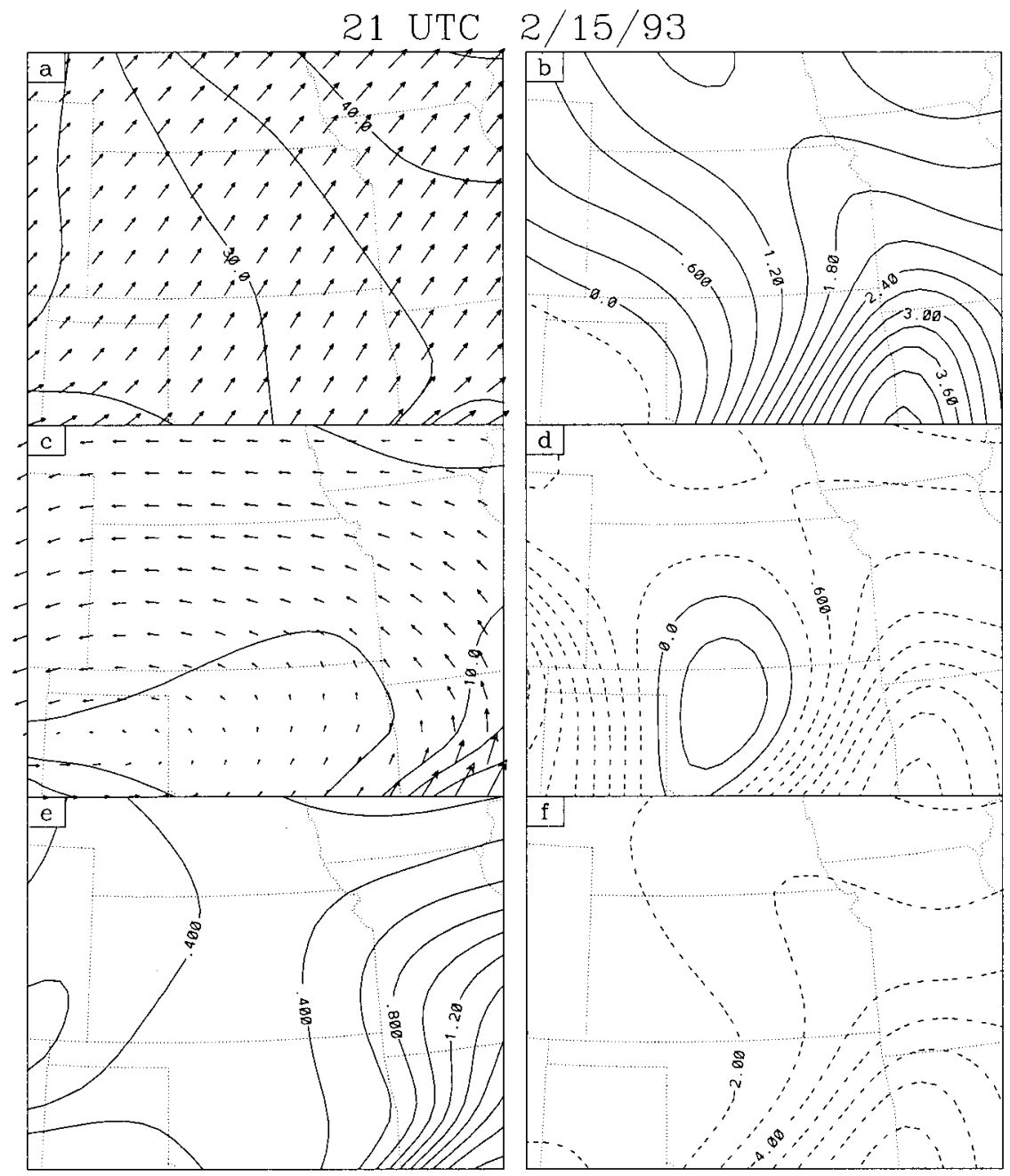

FIG. 11. 2100 UTC 15 Feb 1993 profiler analysis of (a) 300-mb winds and vector magnitude (contour interval of $\left.5 \mathrm{~m} \mathrm{~s}^{-1}\right)$, (b) 300-mb adjusted divergence $\left(\times 10^{5} \mathrm{~s}^{-1}\right.$; contour interval of 0.3 $\mathrm{s}^{-1}$ ), (c) same as (a) except for $800 \mathrm{mb}$, (d) same as (b) except for $800 \mathrm{mb}$, (e) $800-\mathrm{mb}$ virtual temperature advection (contour interval of $0.2 \mathrm{~K} \mathrm{~h}^{-1}$ ), and (f) 500 -mb vertical motion (adjusted kinematic omega; contour interval of $1 \mu \mathrm{b} \mathrm{s}^{-1}$ ). The vector scaling in (c) differs from that in (a).

Precipitation spread into Arkansas and southern Missouri after 0400 UTC 15 February as deep upward motion increased across the area (Fig. 10a). The associated divergence field, showing increasing low-level convergence and upper-level divergence, is presented in Fig. 10b. Precipitation remained light until around 1500 UTC 15 February, when periods of heavy snow and sleet were reported across northern Arkansas and southern Missouri in association with midtropospheric upward motions exceeding $10 \mu \mathrm{b} \mathrm{s}^{-1}$ (Fig. 10a). A pronounced decrease in precipitation coverage and intensity was observed after 0300 UTC 16 February, coincident with the onset of lower-tropospheric subsidence.

Although time-height displays of various fields allow forecasters to diagnose meso- $\alpha$-scale weather patterns over profiler triangles, profiler-derived constant pressure analyses allow forecasters to track these features as well as examine their spatial distribution, both of which offer considerable advantages for real-time applications. In this case, an entrance region of the upper-level jet is evident over the mid-Mississippi Valley at 2100 UTC 15 February, with upper-level divergence values across western Arkansas exceeding $4 \times 10^{-5} \mathrm{~s}^{-1}$ (Figs. 11a,b). The nose of a low-level wind maximum extends into Arkansas (Fig. 11c), where strong low-level convergence (Fig. 11d), warm thermal advection (Fig. 11e), and deep upward motion (Figs. 10a, 11f) exist. Satellite imagery and surface precipitation observations compare favorably with the profiler-derived vertical motion estimates (Fig. 12). The higher cloud tops and heaviest precipitation accumulation occur across portions of eastern Oklahoma, Arkansas, and Missouri, the region of most intense midtropospheric upward motion (Fig. 11f).

By 0300 UTC 16 February, upper-level divergence 
(a)

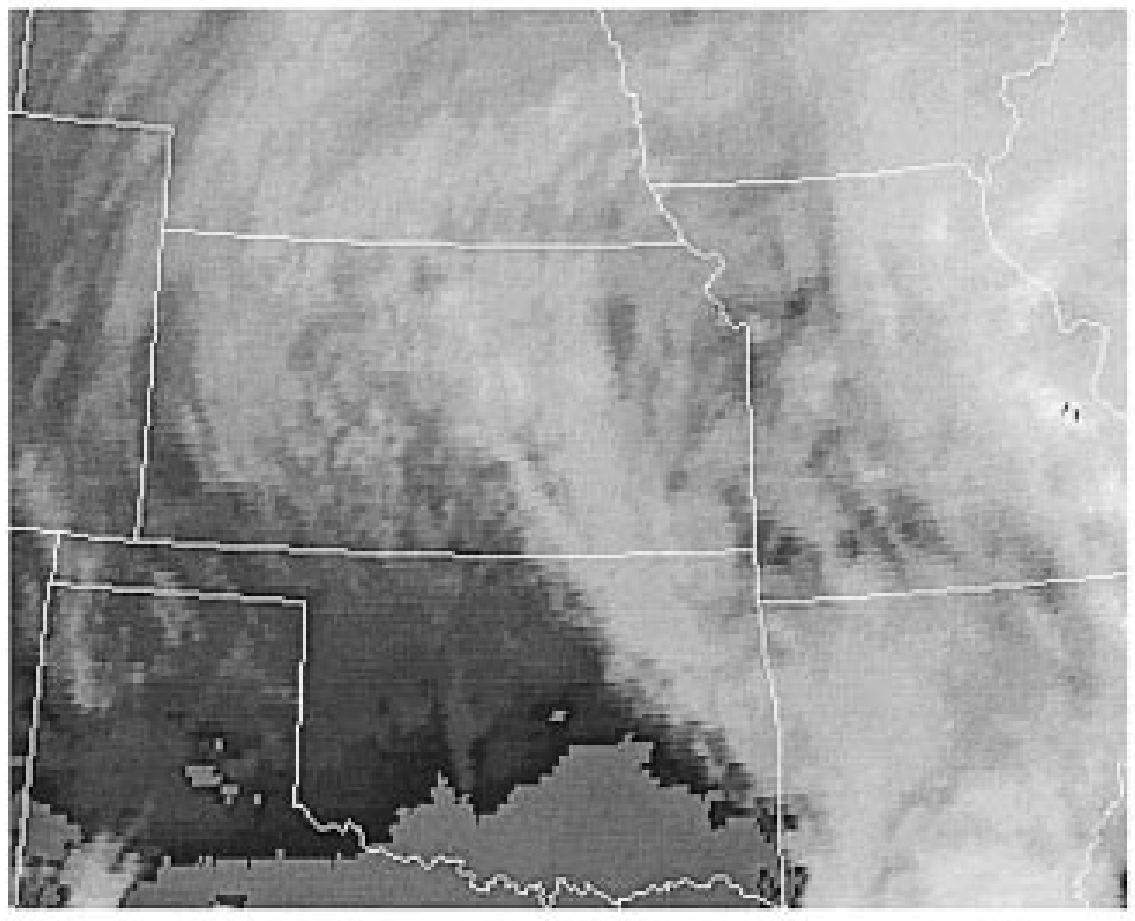

(b) 3-HOURLY PRECIPITATION (mm) ENDING AT 21 UTC 15 FEB 1993

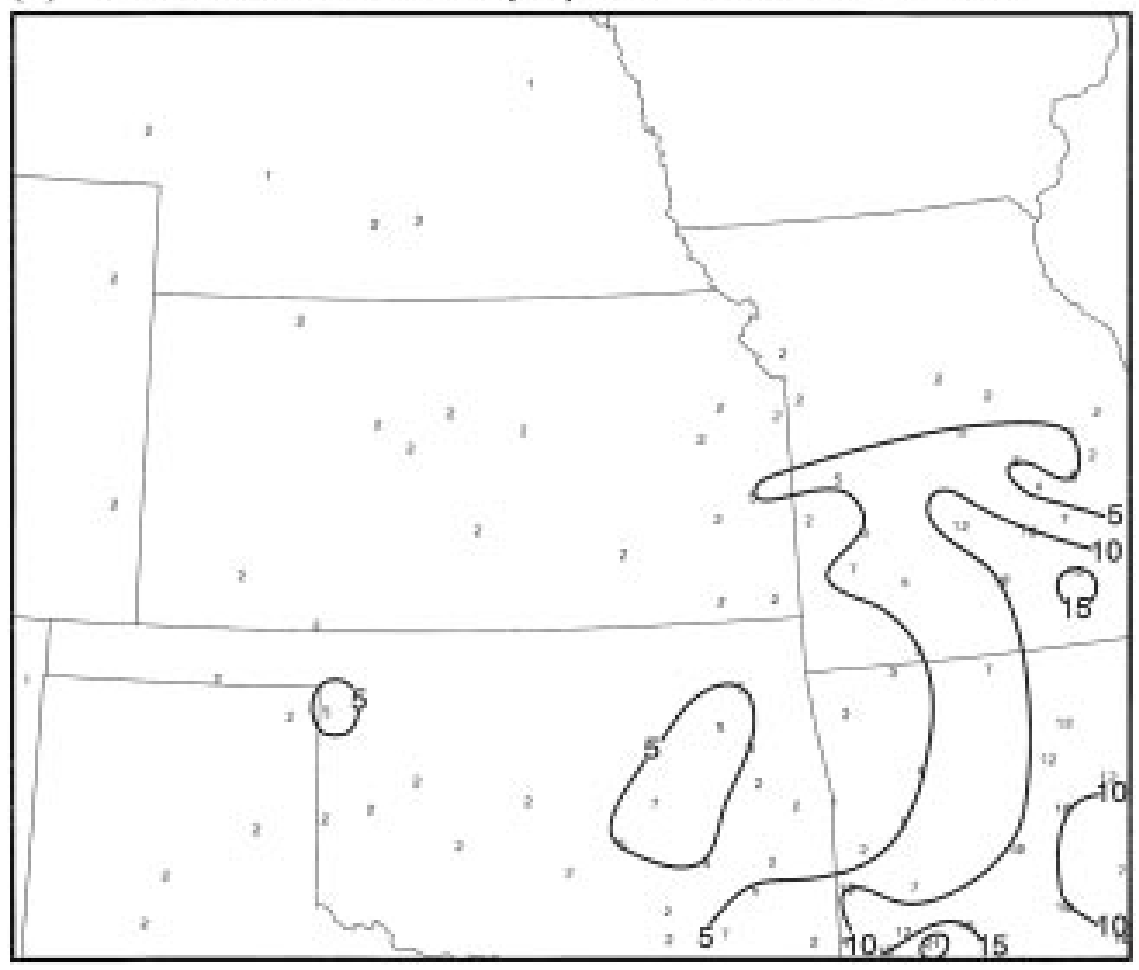

FIG. 12. (a) GOES-7 infrared imagery for 2100 UTC 15 Feb 1993. The image has been enhanced to highlight the colder cloud tops across Arkansas and Missouri and (b) accumulated precipitation $(\mathrm{mm})$ for the 3-h period ending 2100 UTC 15 Feb 1993. Precipitation data are from the hourly precipitation database. 


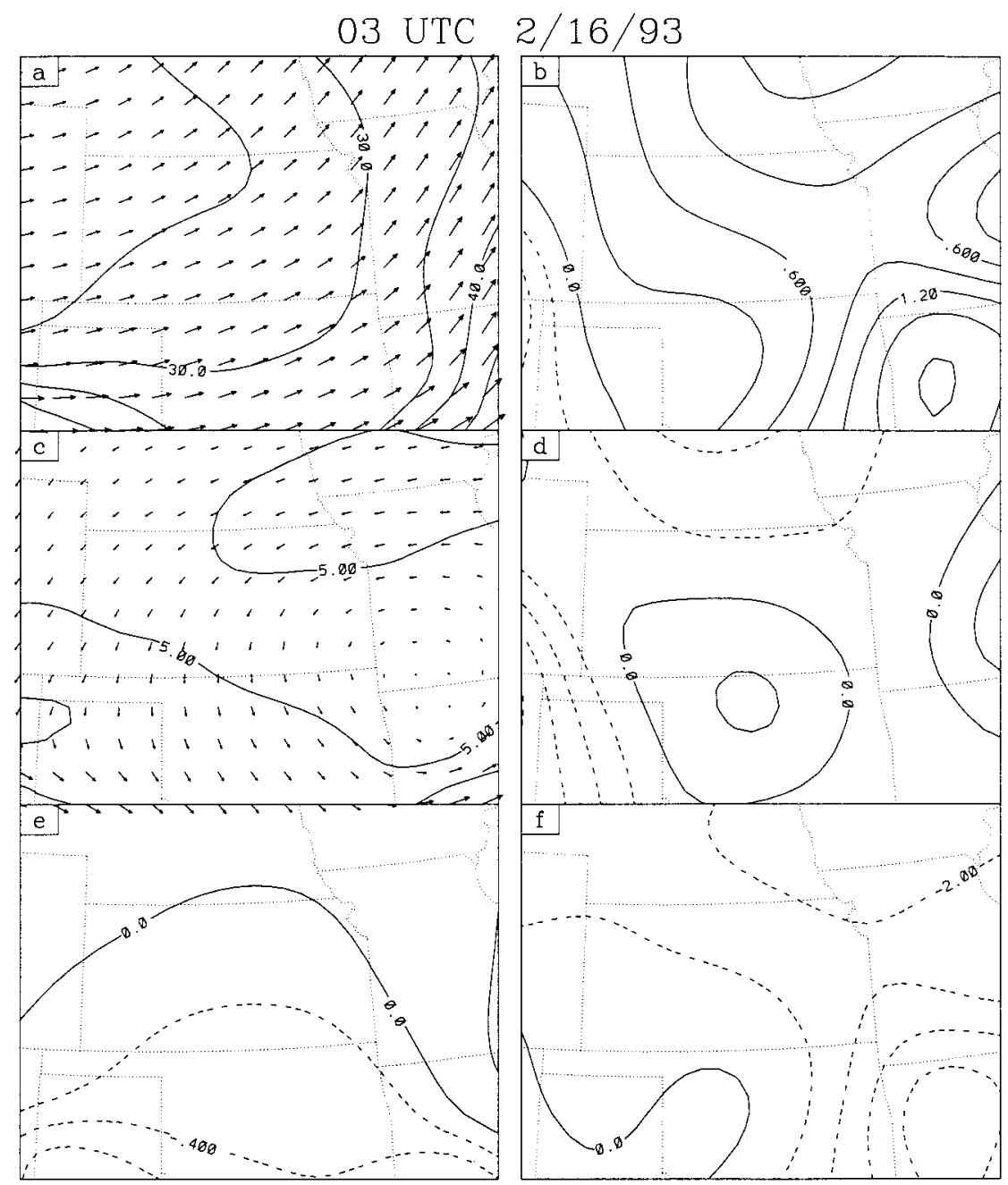

FIG. 13. Same as Fig. 11 except for 0300 UTC 16 Feb 1993.

remained across the southern plains and lower Mississippi Valley, but it had weakened considerably (less than one-half its earlier magnitude) as the entrance region of the jet moved eastward (Figs. 13a,b). Low-level winds became weakly divergent over much of the area (Figs. $13 \mathrm{c}, \mathrm{d})$. Although relatively weak midlevel upward motion persisted (Fig. 13f), the precipitation had mostly ended since the low-level flow was from the northwest, which brought in cooler (Fig. 13e), drier air.

\section{Summary and concluding remarks}

Previous studies have used 2D objective wind analysis procedures whereby data from each profiler station was analyzed independently of data from nearby stations. In this study, we have proposed a 4D objective analysis scheme, such that each station's time-height wind analysis not only incorporates data from its own time series of observations, but neighboring stations' data as well. A primary advantage of the 4D analysis scheme lies in its ability to reduce the negative impacts of significant data voids. Specifically, it has been shown that the unrealistic high-gradient regions within data voids that result from the $2 \mathrm{D}$ technique may be eliminated when neighboring data are incorporated into the analysis scheme. Also, the degree of impact that neighboring data have on station analyses is easily controlled by the appropriate smoothing parameter. The $2 \mathrm{D}$ technique for diagnosing kinematic and thermodynamic structure over profiler triangles has been shown previously to be suitable, however, if no large data gaps exist for those stations involved in the analysis (CSDP95; SCD96).

As a consequence of dealing effectively with large data voids by reducing the negative impacts of missing data, the 4D analysis scheme is able to produce spatially and temporally consistent time-height wind analyses for each station of the WPN. Line integral-equivalent methods then may be used to produce time-height series of kinematic fields over many profiler triangles. Alone, these triangle analyses provide information analogous to that provided by the $2 \mathrm{D}$ technique. However, by com- 
bining information derived from dozens of profiler triangles, hourly analyses detailing the spatial distribution of various parameters are possible. These profiler analyses that provide details concerning the horizontal distribution of kinematic and thermodynamic variables (in addition to the vertical and temporal distribution) provide a more coherent $4 \mathrm{D}$ perspective of weather systems than is derived by the 2D technique. Unfortunately, the limited areal coverage of the WPN precludes the study of weather systems beyond the central United States (such as East Coast cyclogenesis) using multiprofiler techniques such as those described herein. However, across the central United States, such analyses applied to the high temporal resolution profiler data might allow forecasters to improve short-term forecasts not only through profiler diagnostics, but also through improved numerical forecasts when the data are incorporated into a data assimilation system (Smith and Benjamin 1993).

We have chosen to use an objective analysis scheme rather than a data assimilation procedure to generate gridded wind fields for three primary reasons. First, data assimilation techniques typically force some sort of dynamic balance constraint between the wind and mass fields, such as geostrophy. This type of balance may be suitable for large-scale flow patterns, but may be in serious error for the scales of motion resolved by the hourly profiler network. Thus, as Parsons and Dudhia (1997) state, "assimilated fields are directly dependent upon model characteristics and parameterizations." Objective analysis schemes demand no such balance. Data assimilation techniques provide obvious advantages for model initialization, but they are not required—or even necessarily appropriate-for diagnostic analyses such as those presented herein. Second, the response characteristics of the Barnes objective analysis scheme are well known and easily computable, whereas the same cannot be said for data assimilation procedures. Finally, the simplicity of objective analysis schemes for gridding data makes them particularly amenable for diagnostic studies. Parsons and Dudhia (1997) provide a more thorough discussion of the relative merits of objective analysis and data assimilation methods.

Acknowledgments. We wish to thank Joan O'Bannon (NSSL) for assisting with several of the figures, Dave Watson (CSIRO, Perth, Australia) for his assistance with the Delauney triangulation algorithm, NCDC for providing the satellite data, and Daphne Zaras (NSSL) for her assistance in displaying the satellite imagery. We also appreciate the thoughtful comments of David Stensrud (NSSL), Robert Johns (SPC), Bill Collins (NCEP), Eugenia Kalnay (NCEP), and the two anonymous reviews whose comments helped us to clarify and improve the manuscript.

\section{REFERENCES}

Barnes, S. L., 1973: Mesoscale objective map analysis using weighted time-series observations. NOAA Tech. Memo. ERL NSSL-69,
National Severe Storms Laboratory, Norman, OK, 60 pp. [NTIS COM-73-10781.]

_- 1978: Oklahoma thunderstorms on 29-30 April 1970. Part I: Morphology of a tornadic storm. Mon. Wea. Rev., 106, 673684.

_ 1994: Applications of the Barnes objective analysis scheme. Part I: Effects of undersampling, wave position, and station randomness. J. Atmos. Oceanic Technol., 11, 1433-1448.

Bellamy, J. C., 1949: Objective calculations of divergence, vertical velocity and vorticity. Bull. Amer. Meteor. Soc., 30, 45-49.

Bluestein, H. B., and D. A. Speheger, 1995: The dynamics of an upper-level trough in the baroclinic westerlies: Analysis based upon data from a wind profiler network. Mon. Wea. Rev., 123, $2369-2383$.

Brewster, K. A., and T. W. Schlatter, 1986: Automated quality control of wind profiler data. Preprints, 11th Conf. on Weather Forecasting and Analysis, Kansas City, KS, Amer. Meteor. Soc., 171176.

Brown, R. A., and V. T. Wood, 1991: On the interpretation of singleDoppler velocity patterns within severe thunderstorms. Wea. Forecasting, 6, 32-48.

Carlson, C. A., and G. S. Forbes, 1989: A case study using kinematic quantities derived from a triangle of VHF Doppler wind profilers. J. Atmos. Oceanic Technol., 6, 769-778.

Carr, F. H., P. L. Spencer, C. A. Doswell III, and J. D. Powell, 1995: A comparison of two objective analysis techniques for profiler time-height data. Mon. Wea. Rev., 123, 2165-2180.

Ceselski, B. F., and L. L. Sapp, 1975: Objective wind field analysis using line integrals. Mon. Wea. Rev., 103, 89-100.

Davies-Jones, R., 1993: Useful formulas for computing divergence, vorticity, and their errors from three or more stations. Mon. Wea. Rev., 121, 713-725.

Doswell, C. A., III, 1977: Obtaining meteorologically significant surface divergence fields through the filtering property of objective analysis. Mon. Wea. Rev., 105, 885-892.

— sampled vector point functions. J. Atmos. Sci., 45, 242-253.

- and S. G. Lasher-Trapp, 1997: On measuring the degree of irregularity in an observing network. J. Atmos. Oceanic Technol., 14, 120-132.

Hermes, L. G., 1991: Comparisons of rawinsonde-deduced kinematic and thermodynamic quantities with those deduced from VHF profiler observations. Mon. Wea. Rev., 119, 1693-1712.

Karyampudi, V. M., M. L. Kaplan, S. E. Koch, and R. J. Zamora, 1995: The influence of the Rocky Mountains on the 13-14 April 1986 severe weather outbreak. Part I: Mesoscale lee cyclogenesis and its relationship to severe weather and dust storms. Mon. Wea. Rev., 123, 1394-1422.

Koch, S. E., M. DesJardins, and P. J. Kocin, 1983: An interactive Barnes objective map analysis scheme for use with satellite and conventional data. J. Climate Appl. Meteor., 22, 1487-1503.

Menzel, W. P., and J. F. W. Purdom, 1994: Introducing GOES-I: The first of a new generation of Geostationary Operational Environmental Satellites. Bull. Amer. Meteor. Soc., 75, 757-781.

Neiman, P. J., and M. A. Shapiro, 1989: Retrieving horizontal temperature gradients and advections from single-station wind profiler observations. Wea. Forecasting, 4, 222-233.

Parsons, D. B., and J. Dudhia, 1997: Observing system simulation experiments and objective analysis tests in support of the goals of the Atmospheric Radiation Measurement Program. Mon. Wea. Rev., 125, 2353-2381.

Pauley, P. M., and X. Wu, 1990: The theoretical, discrete, and actual response of the Barnes objective analysis scheme for one-and two-dimensional fields. Mon. Wea Rev., 118, 1145-1163.

Ripley, B. D., 1981: Spatial Statistics. Wiley-Interscience, 252 pp.

Schaefer, J. T., and C. A. Doswell III, 1979: On the interpolation of a vector field. Mon. Wea. Rev., 107, 458-476.

Shuman, F. G., 1957: Numerical methods in weather prediction. Part II: Smoothing and filtering. Mon. Wea. Rev., 85, 357-361.

Smith, T. L., and S. G. Benjamin, 1993: Impact of network wind 
profiler data on a 3-h data assimilation system. Bull. Amer. Meteor. Soc., 74, 801-807.

Spencer, P. L., F. H. Carr, and C. A. Doswell III, 1996: Diagnosis of an amplifying and decaying baroclinic wave using wind profiler data. Mon. Wea. Rev., 124, 209-223.

Strauch, R. G., D. A. Merritt, K. P. Moran, K. B. Earnshaw, and D. van de Kamp, 1984: The Colorado wind-profiling network. $J$. Atmos. Oceanic Technol., 1, 37-49.
Yoe, J. G., M. F. Larsen, and E. J. Zipser, 1992: VHF wind-profiler data quality and comparison of methods for deducing horizontal and vertical air motions in a mesoscale convective storm. $J$. Atmos. Oceanic Technol., 9, 713-727.

Zamora, R. J., M. A. Shapiro, and C. A. Doswell III, 1987: The diagnosis of upper tropospheric divergence and ageostrophic wind using profiler wind observations. Mon. Wea. Rev., 115, 871-884. 